\title{
Nouveaux anticoagulants. Mise au point et enjeux en chirurgie orale
}

\author{
Cédric Lansonneur ${ }^{1}{ }^{*}$, Pauline Guillou $^{1}$, Aurélie Hacquard ${ }^{1}$, Manon Devisse $^{1}$, \\ Julie Lelievre ${ }^{1}$, Grégoire Le Gal ${ }^{2}$, Sylvie Boisramé-Gastrin ${ }^{1}$
}

${ }^{1}$ Service d'Odontologie, CHRU/UFR d'Odontologie, Université de Bretagne occidentale, Brest, France

2 Service de Médecine interne, CHRU, Brest, France

(Reçu le 18 septembre 2012, accepté le 24 octobre 2012)

Mots clés : anticoagulants / héparines / dabigatran etexilate / rivaroxaban / fondaparinux / chirurgie orale / hémorragie

Résumé - Les anti-vitamines K (AVK) et les héparines non fractionnées (HNF), accompagnées depuis la fin des années 80 des héparines de bas poids moléculaire (HBPM), ont constitué, pendant des décennies, les thérapeutiques de choix dans la prévention et dans la prise en charge des accidents thromboemboliques. Les anticoagulants parentéraux d'action rapide (HNF et HBPM) sont utilisés dans la phase initiale et les anticoagulants oraux de longue durée d'action (AVK) indiqués pour le traitement au long cours de ces pathologies.

Ces dernières années, les efforts de recherche sur les traitements anticoagulants ont visé à obtenir des bénéfices en termes d'efficacité, de délai d'action, d'affinité de liaison aux facteurs de la coagulation, de surveillance thérapeutique. Pour ce faire, différentes étapes de la cascade de la coagulation ont été ciblées, notamment l'inhibition de la thrombine et l'inhibition du facteur Xa.

Ainsi, le fondaparinux et l'idraparinux sont des pentasaccharides de synthèse dérivés de la portion de l'héparine se liant à l'antithrombine. Celle-ci inhibe fortement le facteur Xa de manière indirecte. Le fondaparinux présente l'avantage de ne pas entraîner de thrombopénie. Il s'administre par une unique injection journalière sous-cutanée. Pour l'idraparinux, il suffit d'une injection par semaine également sous-cutanée. L'action inhibitrice anti-Xa marquée d'une part et la demi-vie supérieure à celle des anticoagulants injectables classiques d'autre part, doivent être prises en compte lors de la programmation d'intervention en chirurgie orale chez ces patients.

Encore plus récemment, deux nouvelles molécules viennent d'être mises sur le marché. Cette nouvelle famille d'antithrombotiques oraux est représentée par un anti-Xa direct (rivaroxaban) et un anti-IIa direct (dabigatran). Ces deux molécules présentent l'avantage de ne pas avoir les inconvénients des différents anticoagulants classiques. Il existe désormais un anticoagulant oral facile à manier qui ne nécessite aucune surveillance ni aucun ajustement thérapeutique. On assiste donc à une révolution dans la prise en charge et la prévention des accidents thromboemboliques qui doit amener à repenser l'approche de ce type de patient en chirurgie orale. En effet, la prévention du risque hémorragique chez les patients sous AVK était jusqu'ici bien codifiée et basée sur l'INR. L'absence d'antagoniste pour ces nouveaux anticoagulants oraux risque probablement de nous amener à modifier profondément notre prise en charge en chirurgie orale?

Key words: anticoagulants / heparins / dabigatran etexilate / rivaroxaban / fondaparinux / oral surgery / hemorrhage

Abstract - New anticoagulants: Updating and issues in oral surgery. Heparins, either unfractionated or lowmolecular-weight (UFH and LMWHs), and vitamin K antagonists (VKA) are currently the anticoagulants of choice for the prevention of postoperative venous thromboembolism (VTE) and for the treatment of acute venous and arterial thromboembolism.

Heparins are rapidly acting parenteral anticoagulants. They are widely used for the prevention and initial shortterm treatment of VTE comprising deep vein thrombosis (DVT) and pulmonary embolism (PE). Two VKA are currently widely used for VTE treatment and prophylaxis in medical and surgical patients.

In recent years, research on anticoagulant therapy aimed to obtain benefits in terms of efficacy, onset of action, binding affinity to coagulation factors, therapeutic monitoring. To do this, different stages of the coagulation

\footnotetext{
*Correspondance : cedric.lans@gmail.com
} 
cascade have been identified, including the inhibition of thrombin and factor Xa. Thus, the heparin pentasaccharide mimetics, fondaparinux and idraparinux are synthetic derivatives of the portion of the heparin binding to antithrombin. It strongly inhibits factor Xa indirectly. Fondaparinux has the advantage of not causing thrombocytopenia. It is administered by a single subcutaneous injection daily. Idraparinux, meanwhile, does not inject once a week also subcutaneously. The anti-Xa inhibitory action marked the one hand and the half-life greater than conventional injectable anticoagulants other hand, must be taken into account when planning intervention in oral surgery in these patients.

More recently, two new molecules are being allowed on the market. This family of new antithrombotic agents is represented by a direct anti-Xa (rivaroxaban) and a direct anti-IIa (dabigatran). These two molecules have the immense property of sweeping disadvantages of different anticoagulants classics. There is now an easy to use oral anticoagulant that there is no monitoring or dose adjustment to implement. The coming revolution in terms of care and prevention of thromboembolic events should lead the dentist to rethink the approach to this type of patient. Indeed, the prevention of bleeding risk in patients on VKA was previously well codified, based on the INR. The absence of antagonist for these new oral anticoagulants may profoundly change our management in oral surgery?

Les indications majeures des anticoagulants sont les suivantes: la prévention primaire et secondaire ainsi que le traitement initial et à long terme des maladies thromboemboliques veineuses (MTEV) - thrombose veineuse profonde (TVP) et embolie pulmonaire (EP) -, la prise en charge initiale des syndromes coronariens aigus, la prévention de l'infarctus du myocarde (IDM) chez les patients en fibrillation auriculaire et enfin le traitement des patients porteurs de valves mécaniques. Les anti-vitamines $\mathrm{K}(\mathrm{AVK})$ et les héparines non fractionnées (HNF) et, depuis la fin des années 80 , les héparines de bas poids moléculaire (HBPM), ont constitué les thérapeutiques de choix utilisées en pratique courante pendant des décennies. Il est à noter que les thromboses veineuses et artérielles constituent les causes majeures de morbidité et de mortalité dans les populations vieillissantes. Ces molécules anticoagulantes représentent, de fait, un enjeu majeur. Ainsi, ces dernières années, les efforts de recherche concernant les traitements anticoagulants ont visé à obtenir des bénéfices en terme d'efficacité, de délais d'action, d'affinité de liaison aux facteurs de la coagulation et de surveillance thérapeutique. Pour ce faire, différentes étapes de la cascade de la coagulation ont été ciblées, notamment l'inhibition de la thrombine et du facteur Xa [1].

Dans un premier temps, cet article revient sur les anticoagulants classiques et leurs limites. Puis, on passe en revue les nouveaux anticoagulants disponibles et les études qui les concernent. La conclusion ouvre une réflexion sur la révolution en cours dans la prise en charge et la prévention des accidents thromboemboliques car elle doit amener à repenser l'approche de ce type de patient en chirurgie orale. En effet, la prévention du risque hémorragique chez les patients sous AVK était basée sur l'INR et bien codifiée dans les recommandations de la Société Francophone de Médecine Buccale et de Chirurgie Buccale [2]. L'absence d'antagonistes pour ces nouveaux anticoagulants oraux risque de nous obliger à modifier profondément la prise en charge en chirurgie orale?

\section{Anticoagulants classiques et leurs limites}

Les AVK tels que la fluindione, l'acénocoumarol, la warfarine agissent par blocage de la carboxylation vitamine $\mathrm{K}$ dépendante des facteurs II, VII, IX et X ; elles diminuent ainsi leur activité enzymatique (Tab. I). Leur administration est orale et ils ont un délai d'action long et lentement réversible. La réversibilité de l'effet anticoagulant des AVK peut être obtenue d'une part, par l'interruption du traitement et d'autre part, par l'administration d'un antidote, la vitamine $K$, qui entraîne la normalisation de l'INR en $24 \mathrm{~h}$ per os et en $12 \mathrm{~h}$ à $16 \mathrm{~h}$ en IV. Pour obtenir une réversibilité immédiate, on doit administrer les facteurs vitamines $K$ dépendants (plasma frais) ou un concentré de complexe de prothrombine [3].

Le cytochrome P450 CYP2C9 et le complexe de la vitamine $K$ époxyde réductase présentent un polymorphisme important responsable d'une réponse individuelle au traitement imprévisible, ce qui nécessite une surveillance régulière de l'INR des patients. Il existe également de nombreuses interactions alimentaires (aliments riches en vitamine $\mathrm{K}$ : brocolis, choux, avocats, etc.) et médicamenteuses, et des variations de l'état physiologique du patient qui rendent souvent l'INR très difficile à stabiliser [3] (Tab. II).

Une récente méta-analyse américaine a montré que les patients en fibrillation auriculaire traités par anticoagulants ne passeraient que $51 \%$ à $63 \%$ du temps dans les limites de leur INR thérapeutique. Le reste du temps, ils courraient tantôt un risque thromboembolique et tantôt un risque hémorragique [4]. Le chiffre de 17000 hospitalisations par an pour 600000 patients sous AVK en France amène à poser la question de l'efficacité réelle de ces traitements contraignants [5].

Les héparines non fractionnées (HNF) et les héparines de bas poids moléculaire (HBPM) agissent par liaison à l'antithrombine III qui est l'inhibiteur physiologique des sérines protéases de la coagulation (IIa, IXa Xa, XIa, XIIa) ; elles en potentialisent l'effet par un facteur 1000 . Les HNF, 
Tableau I. Liste des AVK oraux actuellement commercialisés en France.

Table I. Currently marketed vitamin $\mathrm{K}$ anticoagulants in France.

\begin{tabular}{|c|c|}
\hline Dénomination commune internationale & \multicolumn{1}{|c|}{ Nom de spécialité } \\
\hline Dérivé de l'indanedione & \\
\hline Fluindione & PREVISCAN $^{\circledR} 20 \mathrm{mg}$ \\
\hline Dérivés de la coumarine & \\
Acénocoumarol & SINTROM $^{\circledR} 4 \mathrm{mg}$ \\
Warfarine & MINISINTROM $^{\circledR} 1 \mathrm{mg}$ \\
& COUMADINE $^{\circledR} 2 \mathrm{mg}, 5 \mathrm{mg}$ \\
\hline
\end{tabular}

Tableau II. Médicaments interagissant avec les AVK.

Table II. Drugs interacting with vitamin $K$ anticoagulants.

\begin{tabular}{|c|c|}
\hline $\begin{array}{c}\text { Médicaments potentialisant } \\
\text { les AVK }\end{array}$ & $\begin{array}{c}\text { Médicaments diminuant l'efficacité } \\
\text { des AVK }\end{array}$ \\
\hline AINS & Laxatifs \\
Sulfamides (anti-infectieux ou hypoglycémiants) & Barbituriques \\
Hydantoïne & Carbamazépine \\
Hormones thyroïdiennes & Phénitoïne \\
Métronidazole & Rifampicine \\
Fluconazole & Glucocorticoïdes \\
Allopurinol & Oestrogènes \\
AAP & C \\
\hline
\end{tabular}

Tableau III. HBPM actuellement commercialisées en France. Table III. Low molecular weight heparins marketed in France.

\begin{tabular}{|l|l|}
\hline Nadroparine & $\begin{array}{l}\text { FRAXIPARINE }^{\circledR} \\
\text { FRAXODI }^{\circledR}\end{array}$ \\
Enoxoparine & LOVENOX $^{\circledR}$ \\
Tinzaparine & INNOHEP $^{\circledR}$ \\
Daltéparine & FRAGMINE $^{\circledR}$ \\
Réviparine & CLIVARINE $^{\circledR}$ \\
\hline
\end{tabular}

que ce soit par voie sous-cutanée (Calciparine ${ }^{\circledR}$ ) ou IV (Héparine Sodique ${ }^{\circledR}$ ) présentent donc une activité anti-Xa et anti-IIa indirecte. Les HBPM qui sont issues de la dépolymérisation de l'héparine, présentent principalement une activité anti-Xa indirecte (environ 2 à 6 fois supérieure à leur activité anti-IIa). Il existe un antidote à ces héparines et leurs dérivés: le sulfate de protamine en IV qui se lie à l'héparine pour former un complexe biologiquement inactif. Il agit principalement sur l'activité anti-IIa, son action sur les petits fragments des HBPM ayant une activité anti-Xa est incomplète [3].

Les HBPM constituent le traitement de référence pour la prévention des événements thromboemboliques veineux et pour le traitement curatif des thromboses constituées car elles sont plus efficaces et plus sûres que les HNF. Les héparines et leurs dérivés sont également indiqués pour les thromboses artérielles, l'angor instable, l'infarctus du myocarde, l'angioplastie coronaire, la circulation extracorporelle et pour les patients alités (Tab. III). Les limites de ces héparines et de leurs dérivés résident dans le fait que leur administration ne peut être que parentérale et qu'ils nécessitent un suivi régulier de la numération plaquettaire pour prévenir le risque non négligeable de survenue d'une thrombopénie induite par les héparines (TIH). Il faut également noter que les HBPM sont contre-indiquées chez l'insuffisant rénal en raison du risque de surdosage. Là aussi, la question de l'efficacité de ces molécules est soulevée par des résultats montrant que 10 à $15 \%$ des patients subissant une chirurgie orthopédique majeure développent une thrombose veineuse malgré la prophylaxie par HBPM [6].

Les axes de recherche visent donc à mettre au point un anticoagulant idéal qui permettrait une administration du 
Tableau IV. Classification des anticoagulants. Table IV. Anticoagulants classification.

\begin{tabular}{|c|c|c|c|}
\hline \multirow{4}{*}{ 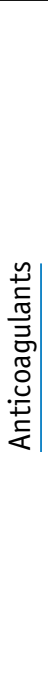 } & \multicolumn{3}{|c|}{$\begin{array}{c}\text { AVK } \\
\text { (inhibant II, VII, IX, X) }\end{array}$} \\
\hline & \multirow{2}{*}{$\begin{array}{l}\text { Inhibiteurs } \\
\text { du facteur X }\end{array}$} & $\begin{array}{l}\text { Anti-Xa indirects } \\
\text { (liant l'antithrom- } \\
\text { bine) }\end{array}$ & $\begin{array}{c}\text { Héparines } \\
\text { HBPM } \\
\text { Oligosaccharides } \\
\text { (Fondaparinux, } \\
\text { idraparinux) }\end{array}$ \\
\hline & & $\begin{array}{l}\text { Anti-Xa direct } \\
\quad=\text { xabans }\end{array}$ & $\begin{array}{c}\text { Rivaroxaban } \\
\text { Edoxaban } \\
\text { Apixaban } \\
\text { Betrixaban } \\
\text { Otamixaban }\end{array}$ \\
\hline & $\begin{array}{l}\text { Inhibiteurs de la } \\
\text { thrombine } \\
\text { (Anti-IIa directs) }\end{array}$ & $\begin{array}{r}\text { Dabic } \\
\text { Argat } \\
\text { Melag } \\
\text { Ximela }\end{array}$ & $\begin{array}{l}\text { atran } \\
\text { oban } \\
\operatorname{tran}^{\ddagger} \\
\operatorname{atran} \ddagger\end{array}$ \\
\hline
\end{tabular}

produit per os et parentérale, une surveillance thérapeutique simple, une fenêtre thérapeutique large, une demi-vie d'élimination appropriée, un délai d'action rapide, une réversibilité d'action rapide et l'absence d'interactions alimentaires ou médicamenteuses.

\section{Nouveaux anticoagulants}

Différentes classifications des nouveaux anticoagulants existent, soit en fonction du facteur sur lequel ils agissent, soit selon leur mode d'action direct ou indirect (Tab. IV). La classification retenue a été établie en fonction de leur mode d'action par rapport à l'antithrombine :

Nouveaux agents antithrombine-dépendants :

HBPM : (certains auteurs les classent parmi les nouveaux anticoagulants)

Pentasaccharides : fondaparinux (Arixtra $\left.{ }^{\circledR}\right)$, idraparinux

Nouveaux agents antithrombine-indépendants :

Anti-Xa directs: rivaroxaban (Xarelto $\left.{ }^{\circledR}\right)$, apixaban, edoxaban

Anti-IIa directs : dabigatran (Pradaxa $\left.{ }^{\circledR}\right)$, argatroban, ximelagatatran.

\section{Inhibiteurs indirects du Xa : pentasaccharides (ou oligosaccharides)}

Les pentasaccharides sont des molécules de synthèse ayant une action indirecte en se liant à l'antithrombine III et en potentialisant ses effets. La fixation à l'antithrombine III étant réversible, une refixation peut se faire à 2 à 3 reprises, ce qui explique que ces produits sont beaucoup plus puissants que les héparines et leurs dérivés. L'autre particularité de ces pentasaccharides de synthèse est l'absence de résidus glycosaminoglycanes permettant la liaison à la thrombine, ce qui leur confère une activité anti-Xa exclusive.

Il existe deux molécules ayant reçu une AMM : le fondaparinux (Arixtra ${ }^{\circledR}, A M M$ en 2004 ) ayant une demi-vie d'élimination de 15 à $20 \mathrm{~h}$ (posologie : 1 injection sous-cutanée/j) et l'idraparinux ayant une demi-vie d'élimination de 5,5 jours (posologie : 1 injection sous-cutanée/semaine). Ces pentasaccharides qui ont une élimination urinaire sous une forme inchangée, n'induisent pas de thrombopénie [7]. Le fondaparinux a reçu une $A M M$ pour les indications suivantes : la prévention primaire des MTEV en chirurgie orthopédique (prothèse totale de genou - PTG - et prothèse totale de hanche - PTH - [8]) le traitement des MTEV (étude Matisse) [9], la prévention primaire des MTEV chez les patients médicaux hospitalisés (étude Artémis) [10] et le traitement aigu de l'angor instable ou de l'IDM avec et sans sus-décalage du segment ST (étude 0asis-6) [11], dernière extension récente de son AMM.

\section{Inhibiteurs directs du $\mathrm{Xa}$}

Le facteur Xa est ciblé par ces nouveaux anticoagulants à action directe car il se situe à la convergence des voies extrinsèque et intrinsèque de la cascade de la coagulation. L'importance de cibler ce facteur réside encore dans le fait qu'une seule molécule de facteur $X a$ génère plus de 1000 molécules de thrombine [12]. Le chef de file de ces nouveaux inhibiteurs directs du Xa est le rivaroxaban commercialisé sous le nom de spécialité Xarelto ${ }^{\circledR}$. C'est un inhibiteur direct compétitif réversible du facteur $X a$. Son administration se fait per os à raison d'une seule prise par jour. Il a une demi-vie d'élimination de 5 à $13 \mathrm{~h}$ et il est éliminé par le rein sous forme inchangée.

Quatre études cliniques de phase III ont été réalisées pour évaluer l'efficacité et la sécurité d'emploi du rivaroxaban par rapport à l'énoxoparine dans la prévention des événements thromboemboliques veineux après chirurgie orthopédique pour PTH ou PTG. Ces quatre études appartenaient au programme RECORD (REgulation of Coagulation in ORthopedic surgery to prevent DVT and PE) et elles ont évalué plus de 12770 patients. Ce sont des essais multinationaux, randomisés et réalisés en double-aveugle. Sur la base de ces études, la commission de transparence de la HAS juge le service médical rendu par le rivaroxaban important. Cependant, l'amélioration du service médical rendu reste mineure en termes d'efficacité par rapport à l'énoxoparine pour la prévention des événements thromboemboliques veineux chez les patients devant subir une intervention pour une PTH ou PTG [13].

Actuellement, la prévention primaire des MTEV en chirurgie orthopédique (PTG et PTH) (études RECORD 1-2-3-4) [14-16] 
Tableau V. Résumé des caractéristiques pharmacologiques des différents anticoagulants. Table V. Summary of the pharmacological characteristics of different anticoagulants.

\begin{tabular}{|c|c|c|c|c|c|}
\hline & $\begin{array}{c}\text { HNF et HBPM } \\
\text { Anti-Xa et IIa indirects }\end{array}$ & AVK & $\begin{array}{c}\text { Fondaparinux } \\
\text { Anti-Xa indirect }\end{array}$ & $\begin{array}{c}\text { Dabigatran } \\
\text { Anti-IIa direct }\end{array}$ & $\begin{array}{c}\text { Rivaroxaban } \\
\text { Anti-Xa direct }\end{array}$ \\
\hline Administration & parentérale & orale & parentérale & orale & orale \\
\hline $\begin{array}{c}\text { Surveillance biologique } \\
+/-\end{array}$ & +++ & rare & non & non \\
\hline $\begin{array}{c}\text { Apparition/ disparition } \\
\text { de l'activité }\end{array}$ & immédiate par voie IV & 3 jours & $2 \mathrm{~h} / 24 \mathrm{~h}$ & $1 \mathrm{~h} 30 / 12 \mathrm{~h}$ & $2 \mathrm{~h} / 12 \mathrm{~h}$ \\
\hline Demi-vie & $\begin{array}{c}\text { HNF }: 60 \text { à } 90 \text { min } \\
\text { HBPM }: 120 \text { à } 180 \text { min }\end{array}$ & 24 à $72 \mathrm{~h}$ & $17 \mathrm{~h}$ à $21 \mathrm{~h}$ & $12 \mathrm{~h}$ à $14 \mathrm{~h}$ & $5 \mathrm{~h}$ à $13 \mathrm{~h}$ \\
\hline $\begin{array}{c}\text { oui } \\
\text { Antidote spécifique }\end{array}$ & $\begin{array}{c}\text { oui } \\
\text { our HBPM })\end{array}$ & non & non & non \\
\hline
\end{tabular}

constitue la seule indication pour laquelle le rivaroxaban a reçu une AMM en 2008. Mais il y a plusieurs études en cours : une étude de phase III pour le traitement des MTEV (étude EINSTEIN-DVT) [14] et pour la prévention des AVC chez les patients en FA d'origine non valvulaire (étude ROCKET-AF) [15], et une étude de phase II pour la prévention secondaire des syndromes coronariens aigus (étude ATLAS) [16].

\section{Inhibiteurs directs de la thrombine}

Contrairement à l'héparine qui inhibe indirectement la thrombine de manière incomplète, de nouveaux anticoagulants anti-IIa ont été développés pour agir directement sur la thrombine. Le facteur IIa est toujours ciblé car il reste l'effecteur final de la cascade de la coagulation convertissant le fibrinogène circulant en fibrine. Il est également l'activateur physiologique le plus important des plaquettes et réalise surtout l'amplification de sa propre génération par rétrocontrôle positif sur les facteurs V, VIII et IX [12] :

Le chef de file de ces nouveaux inhibiteurs directs de la thrombine est le dabigatran commercialisé sous la spécialité Pradax ${ }^{\circledR}$. C'est un inhibiteur direct compétitif réversible de la thrombine. Son administration se fait per os à raison d'une seule prise par jour. Sa demi-vie d'élimination est de 12 à $14 \mathrm{~h}$ et son élimination est rénale sous forme inchangée (Tab. V).

Le dabigatran a reçu son AMM en 2008 pour la prévention primaire des MTEV en chirurgie orthopédique (PTG et PTH) (Etudes RE-MODEL et RE-NOVATE) [17]. Il est actuellement en étude de phase III pour le traitement des MTEV (Etudes RE-COVER, RE-MEDY et RE-SOLVE) ([18] et pour la prévention des AVC chez patients en FA non valvulaire (Etude RE-LY) [19]. C'est d'ailleurs sur cette base que la FDA et Santé Canada ont récemment approuvé le dabigatran etexilate dans cette indication. Il est également en étude de phase II dans la prévention secondaire des syndromes coronariens aigus
(Etude RE-DEEM) ainsi que dans la prévention de l'IDM chez patients en FA non valvulaire (Etude PETRO).

D'après la HAS, le fondaparinux, le dabigatran etexilate et le rivaroxaban n'apportent donc pas de réelle amélioration du service médical rendu en termes de prévention et de traitement de la maladie thromboembolique veineuse par rapport à l'énoxoparine. Ces nouveaux traitements présentent cependant quelques avantages par rapport aux traitements existants comme le montre le Tableau VI, avec notamment un effet anticoagulant prévisible, des interactions alimentaires et médicamenteuses limitées et une administration par voie orale pour le dabigatran etexilate et le rivaroxaban.

Le service médical rendu et le confort d'utilisation apportés par ces nouvelles molécules ne seraient être remis en doute. Cependant, seul le risque hémorragique majeur a été évalué comme critère de tolérance dans les études menées sur ces produits (ACUITY).

\section{Questions soulevées en chirurgie orale}

Ces nouvelles molécules anticoagulantes reposent la question de la gestion du risque hémorragique en chirurgie orale qui était bien codifiée dans les recommandations de la SFMBCB pour la prise en charge des patients sous AVK ; les examens biologiques à réaliser et les protocoles d'hémostase locale à mettre en place en fonction du type d'anticoagulation sont clairement précisés [2]. La gestion des patients sous AVK au long cours devant subir une intervention de chirurgie orale dépend principalement de la valeur de l'INR avant l'intervention. L'arbre décisionnel présenté dans ces recommandations oriente soit vers une hémostase de niveau 1 (hémostase locale, sutures, acide tranexamique) praticable en ville, soit vers une hémostase de niveau 2 (hémostase locale, sutures, acide tranexamique, colle biologique) nécessitant une prise 
Tableau VI. Comparaison des nouveaux anticoagulants avec les caractéristiques de l'anticoagulant idéal [9]. Table VI. Comparison of new anticoagulants with the characteristics of the ideal anticoagulant [9].

\begin{tabular}{|c|c|c|c|}
\hline & Fondaparinux & Dabigatran & Rivaroxaban \\
\hline $\begin{array}{l}\text { Effet anticoagulant prévisible } \\
\text { (sans nécessité de monitoring) }\end{array}$ & oui & oui & oui \\
\hline Bon rapport efficacité / sécurité & oui & oui & oui \\
\hline Court délai d'action à l'initiation et à l'arrêt du traitement & oui & oui & oui \\
\hline Disponibilité d'un antidote & non & non & non \\
\hline Absence d'effets indésirables & non & non & non \\
\hline Interactions alimentaires et médicamenteuses limitées & oui & oui & oui \\
\hline Administration par voie orale & non & oui & oui \\
\hline
\end{tabular}

Tableau VII. Recommandations pour la prise en charge des patients sous traitement anti-vitamines K en chirurgie bucco-dentaire (SFMBCB, 2006).

Table VII. Recommendations for the management of patients on vitamin K antagonists in oral surgery (SFMBCB, 2006).

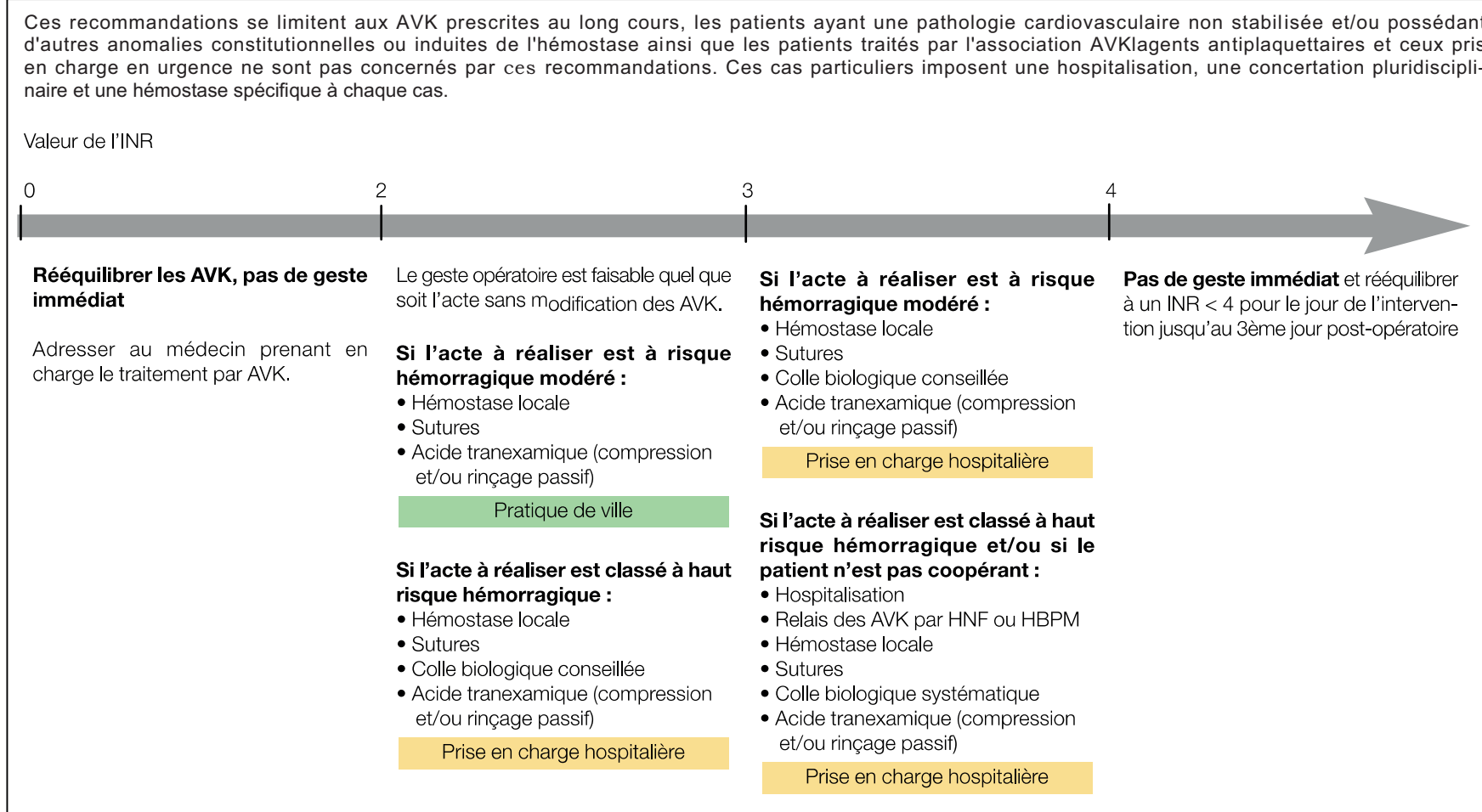

en charge hospitalière car la colle biologique est un produit dérivé du sang (Tab. VII).

Enfin, les nombreuses études de phases II et III concernant ces nouveaux anticoagulants soulèvent une autre question : quelle sera demain la population cible de ces molécules ?

\section{Risque hémorragique avec ces nouveaux anticoagulants ?}

Il n'existe aucun consensus concernant la classification des complications hémorragiques dans les différentes études concernant ces nouveaux produits anticoagulants (ACUIY, TIMI, GUST0, etc.). Cette absence de définition consensuelle est problématique pour la comparaison des critères choisis dans les études cliniques sur les traitements prophylactiques des évènements thromboemboliques veineux. Ainsi, seul le risque hémorragique majeur a été évalué comme critère de tolérance dans les études menées sur ces produits.

La revue de littérature permet de retrouver des hémorragies majeures: hémorragies intracrânienne, rétro-péritonéale, 
intraoculaire, hémorragies exigeant une intervention radiologique ou chirurgicale pour accéder au site, hématomes $\geq$ à $5 \mathrm{~cm}$ au site de ponction, hémorragies entrainant une chute de la concentration en hémoglobine $\geq 4 \mathrm{~g} / \mathrm{dL}$ sans source manifeste de saignement, hémorragies entrainant une chute de la concentration en hémoglobine $\geq 3 \mathrm{~g} / \mathrm{dL}$ avec source manifeste de saignement, ré-intervention pour saignement, hémorragies nécessitant la transfusion de sang ou de produits sanguins $>$ 2 culots $[12,20]$. Mais qu'en est-t-il des hémorragies mineures engendrées par ces produits ? Certains auteurs comme Turpie et al. (2002) suggèrent que les nouveaux anticoagulants sont de nature à réduire le risque de survenue d'hémorragie majeure lors de la chirurgie orthopédique alors qu'ils augmentent le risque d'hémorragies mineures par rapport aux HBPM. Les saignements mineurs sont observés de façon fréquente $(\geq 1 / 10)$, les saignements majeurs de façon moins fréquente $(\geq 1 / 100$ et $<1 / 10$ ) [21].

Les hémorragies mineures ne sont donc définies que par défaut : elles n'appartiennent pas aux hémorragies majeures. Est-ce l'absence de consensus sur la définition des hémorragies mineures qui est responsable de la non évaluation du risque hémorragique en chirurgie orale pour ces nouveaux anticoagulants?

\section{Absence de surveillance biologique}

L'usage des nouveaux anticoagulants est limité par l'absence de tests de laboratoire de routine faciles à mettre en œuvre et peu onéreux pour mesurer l'activité du produit et optimiser le rapport bénéfice-sécurité [20]. Les agents antiXa directs (rivaroxaban et apixaban) ont des effets variables et transitoires sur le temps de prothrombine (TP). La mesure de l'activité du facteur Xa est à l'étude mais elle n'est pas non encore standardisée [22].

Les agents anti-IIa directs (dabigatran) ne peuvent être monitorés que de manière indirecte par le temps de thromboplastine partiellement activée (aPTT) ou TCE (temps de coagulation à l'écarine) qui serait plus linéaire mais qui est moins répandu $[20,23]$. La limite de ce test réside dans le fait que le aPTT atteint un plateau à forte concentration. Le temps de dissolution du caillot est plus linéaire mais moins répandu.

Bien que la surveillance de la coagulation ne soit pas nécessaire en routine avec les nouveaux anticoagulants, on voit que, pour monitorer correctement l'activité de ces nouvelles molécules, des investigations et des recherches sont absolument nécessaires [12]. Dans certaines circonstances, on devrait pouvoir s'assurer de la disparition des effets anticoagulants du traitement, notamment avant une intervention chirurgicale ou encore en cas d'accident hémorragique ou thrombotique. Par ailleurs, le monitorage périodique permet de s'assurer de la compliance du patient au traitement [12,22]. Certains auteurs comme Buller et al. font remarquer qu'en l'absence de moyens de surveillance biologique efficace, une accumulation du produit peut aboutir à des accidents hémorragiques majeurs [14].

L'évaluation du risque hémorragique avec les nouveaux anticoagulants repose, pour le moment, uniquement sur des critères pharmacologiques tels que la dose administrée, la demi-vie du produit, le délai d'administration après l'intervention chirurgicale et la première dose administrée [5].

\section{Facteurs de risque associés}

Pour ces nouveaux anticoagulants, les études de tolérance ont permis de mettre en évidence les facteurs de risques hémorragiques suivants:

- sujet âgé,

- sujet de faible poids $(<50 \mathrm{~kg})$,

- insuffisance rénale.

Certains auteurs insistent sur la nécessité de surveiller la fonction rénale pour éviter tout surdosage car la plupart des nouveaux anticoagulants sont éliminés pour partie par le rein sous forme inchangée [24]. Ainsi, le dabigatran nécessite un ajustement de dose chez les patients présentant une insuffisance rénale modérée et est contre-indiqué chez les patients présentant une insuffisance rénale sévère. Le rivaroxaban peut être administré à dose fixe pour la prévention des accidents thromboemboliques veineux chez les patients présentant une insuffisance rénale modérée et doit être utilisé avec prudence chez les patients présentant une insuffisance rénale sévère. L'excrétion de l'apixaban est aussi en partie dépendante de la fonction rénale; cependant, l'impact de l'insuffisance rénale n'a pas été déterminé. Des données supplémentaires sont attendues concernant l'utilisation de ces nouveaux anticoagulants en cas d'insuffisance rénale [25].

- insuffisance hépatique sévère,

- traitements intercurrents susceptibles d'accroître le risque hémorragique (AINS, etc.)

\section{Absence d'antidotes}

Ces nouvelles molécules anticoagulantes ne posséde pas d'antidote. Certes, leurs demi-vies courtes limitent le besoin en antidote. Cependant, en cas de nécessité de stopper immédiatement l'effet de ces molécules, l'absence d'antidote peut s'avérer problématique. Quelques pistes ont été évoquées. Ainsi, du fait de sa faible liaison aux protéines, le dabigatran pourrait être éliminé par dialyse ou hémofiltration. Pour l'ensemble des nouveaux anticoagulants oraux, des résultats précliniques encourageants ont été obtenus avec l'utilisation de facteur VIIa recombinant ou de concentrés de complexe de prothrombine activée [26]. De même, des études récentes chez l'animal ont confirmé l'efficacité d'un facteur Xa mutant recombinant susceptible d'annihiler les effets anticoagulants des inhibiteurs du Xa. Ce facteur Xa mutant recombinant présente un site actif bloqué qui n'a pas de domaine de liaison 
membranaire. Il entre donc en compétition avec le facteur $X a$ endogène pour l'inhibiteur. Cela constitue une piste de développement d'un antidote pour les anti-Xa directs [12].

Prise en charge des hémorragies majeures des patients sous nouveaux anticoagulants

La revue de littérature a mis en évidence quelques pistes concernant la gestion des hémorragies majeures chez les patients traités par ces nouveaux anticoagulants : l'arrêt de l'anticoagulant, la reprise chirurgicale (radioscopie ou endoscopie interventionnelle), l'utilisation d'agents hémostatiques comme un antifibrinolytique (acide tranexamique) ou de la desmopressine (Minirin ${ }^{\circledR}$ ) ou encore de facteur VIIa recombinant (Novoseven ${ }^{\circledR}$ ) et enfin, la technique d'épuration mécanique de l'agent anticoagulant (dialyse, hémoperfusion et/ou plasmaphérèse) $[20,27]$.

Évolution de la population cible pour ces nouveaux anticoagulants

Actuellement, les indications des nouveaux anticoagulants dont les AMM ont été validées sont encore limitées : il s'agit principalement de la thromboprophylaxie péri-opératoire et du traitement curatif des TVP aiguës et de l'embolie pulmonaire. Cela représente environ 90000 à 140000 patients par an en France. En pratique de ville, les chirurgiens oraux ne sont guère concernés pour l'instant. Or, l'administration orale et l'absence de monitorage pour ces drogues les rendent particulièrement adaptées à une utilisation en ambulatoire et à l'application des recommandations qui visent à étendre le traitement prophylactique aux patients à risque thrombotique [12].

L'évolution se fait vers un élargissement des indications et un allongement de la durée des traitements notamment en cardiologie, avec la fibrillation auriculaire et le traitement des patients coronariens où le traitement se ferait à vie [5].

\section{Conclusion}

Cet article a pour but d'attirer l'attention sur ces nouvelles molécules anticoagulantes et le service médical rendu. Leur usage est actuellement assez restreint et principalement hospitalier mais, avec le temps, on va probablement assister à un élargissement des indications et à une augmentation de la durée des traitements.

Le risque hémorragique lié à ces molécules sans antagoniste efficace est réel. Il semble donc opportun de rechercher ces molécules lors de l'interrogatoire (en particulier en milieu hospitalier) et d'évaluer les facteurs de risque hémorragique associés. Pour l'instant, il n'existe pas dans la littérature d'évaluation du risque hémorragique en chirurgie orale pour les patients traités avec ces nouveaux anticoagulants. L'absence de tests de laboratoire pertinents pour mesurer l'efficacité du traitement et déterminer le risque hémorragique, amène à poser la question du niveau de prise en charge et de la mise en place éventuelle de moyens spécifiques pour traiter ces patients. Ainsi, la comparaison entre le protocole d'hémostase de niveau 1 pour les patients sous AVK (éponges hémostatiques intraalvéolaires, sutures résorbables en points séparés et compression avec des compresses imbibées d'acide tranexamique) et le protocole d'hémostase de niveau 2 (éponges hémostatiques intra-alvéolaires, sutures résorbables en points séparés, compression avec des compresses imbibées d'acide tranexamique et utilisation de colle biologique) lors d'une extraction dentaire simple chez des patients traités par ces nouveaux anticoagulants pourrait être intéressante. La question du coût de la prise en charge globale de ce type de patient traité par nouveaux anticoagulants est posée.

\section{Conflits d'intérêt : aucun}

\section{Références}

1. Haas S, Schellong S. New anticoagulants: from bench to bedside. Hamostaseologie 2007;27:41-7.

2. SFMBCB. Recommandations pour la prise en charge des patients sous traitement anti-vitamines $\mathrm{K}$ en chirurgie bucco-dentaire. Méd buccale Chir buccale 2006;12:188-92.

3. Levi MM, Eerenberg E, Lowenberg E, Kamphuisen PW. Bleeding in patients using new anticoagulants or antiplatelet agents: risk factors and management. Neth J Med 2010;68:68-76.

4. Baker WL, Cios DA, Sander SD, Coleman CI. Meta-analysis to assess the quality of warfarin control in atrial fibrillation patients in the United States. J Manag Care Pharm 2009;15:244-52.

5. Albaladejo P. Synthèse et perspectives (Rivaroxaban). Ann $\mathrm{Fr}$ Anesth Rea 2008;27Suppl3:S28-31.

6. Strebel N, Prins M, Agnelli G, Buller HR. Preoperative or postoperative start of prophylaxis for venous thromboembolism with low-molecular-weight heparin in elective hip surgery? Arch Intern Med 2002;162:1451-6.

7. Lobo B, Finch C, Howard A, Minhas S. Fondaparinux for the treatment of patients with acute heparin-induced thrombocytopenia. Thromb Haemost 2008;99:208-14.

8. Turpie AG, Eriksson BI, Lassen MR, Bauer KA. A meta-analysis of fondaparinux versus enoxaparin in the prevention of venous thromboembolism after major orthopaedic surgery. J South Orthop Assoc 2002;11:182-8.

9. McRae SJ, Ginsberg JS. The diagnostic evaluation of deep vein thrombosis. Am Heart Hosp J 2004;2:205-10.

10. Cohen AT, Davidson BL, Gallus AS, Lassen MR, Prins MH, Tomkowski W, Turpie AG, Egberts JF, Lensing AW. Efficacy and safety of fondaparinux for the prevention of venous thromboembolism in older acute medical patients: randomised placebo controlled trial. Br Med J 2006;332:325-9.

11. Wienbergen $\mathrm{H}$, Zeymer $U$. Management of acute coronary syndromes with fondaparinux. Vasc Health Risk Manag 2007;3:321-9. Epub 2007/08/21. 
12. Weitz JI. New oral anticoagulants in development. Thromb Haemost 2010;103:62-70.

13. HAS. Commission de transparence. Rivaroxaban 2009.

14. Buller HR, Davidson BL, Decousus H, Gallus A, Gent M, Piovella F, Prins MH, Raskob G, Segers AE, Cariou R, Leeuwenkamp 0, Lensing AW. Fondaparinux or enoxaparin for the initial treatment of symptomatic deep venous thrombosis: a randomized trial. Ann Intern Med 2004;140:867-73.

15. Rivaroxaban-once daily, oral, direct factor Xa inhibition compared with vitamin $\mathrm{K}$ antagonism for prevention of stroke and Embolism Trial in Atrial Fibrillation: rationale and design of the ROCKET AF study. Am Heart J 2010;159:340-7.

16. Mega JL, Braunwald E, Mohanavelu S, Burton P, Poulter R, Misselwitz F, Hricak V, Barnathan ES, Bordes P, Witkowski A, Markov V, Oppenheimer L, Gibson CM. Rivaroxaban versus placebo in patients with acute coronary syndromes (ATLAS ACSTIMI 46): a randomised, double-blind, phase II trial. Lancet 2009;374:29-38.

17. Eriksson $B I$, Dahl $O E$, Rosencher $N$, Kurth $A A$, van Dijk $C N$, Frostick SP, Prins MH, Hettiarachchi R, Hantel S, Schnee J, Buller HR. Dabigatran etexilate versus enoxaparin for prevention of venous thromboembolism after total hip replacement: a randomised, double-blind, non-inferiority trial. Lancet 2007;370:949-56.

18. Schulman S, Kearon C, Kakkar AK, Mismetti P, Schellong S, Eriksson H, Baanstra D, Schnee J, Goldhaber SZ. Dabigatran versus warfarin in the treatment of acute venous thromboembolism. New Eng J Med 2009;361:2342-52.

19. Ezekowitz MD, Connolly S, Parekh A, Reilly PA, Varrone J, Wang S, Oldgren J, Themeles E, Wallentin L, Yusuf S. Rationale and design of RE-LY: randomized evaluation of long-term anticoagulant therapy, warfarin, compared with dabigatran. Am Heart J 2009;157:805-10.

20. Crowther MA, Warkentin TE. Bleeding risk and the management of bleeding complications in patients undergoing anticoagulant therapy: focus on new anticoagulant agents. Blood 2008;111:4871-9.

21. Turpie AG, Bauer KA, Eriksson BI, Lassen MR. Fondaparinux vs enoxaparin for the prevention of venous thromboembolism in major orthopedic surgery: a meta-analysis of 4 randomized double-blind studies. Arch Intern Med 2002;162:1833-40.

22. Samama CM. Thromboprophylaxie perioperatoire : brève revue et recommandations. Ann Fr Anesth Rea 2008;27Suppl3:S2-8.

23. Stangier J, Rathgen K, Stahle H, Gansser D, Roth W. The pharmacokinetics, pharmacodynamics and tolerability of dabigatran etexilate, a new oral direct thrombin inhibitor, in healthy male subjects. Br J Clin Pharmacol 2007;64:292-303.

24. Phillips KW, Ansell J. The clinical implications of new oral anticoagulants: will the potential advantages be achieved? Thromb Haemost 2010;103:34-9.

25. Harder S. Renal profiles of anticoagulants. J Clin Pharmacol 2012;52:964-75.

26. Perzborn E, Kubitza D, Misselwitz F. Rivaroxaban - A novel, oral, direct factor $X a$ inhibitor in clinical development for the prevention and treatment of thromboembolic disorders. Hamostaseologie 2007;27:282-9.

27. Huvers F, Slappendel R, Benraad B, van Hellemondt G, van Kraaij $M$. Treatment of postoperative bleeding after fondaparinux with rFVIIa and tranexamic acid. Neth J Med 2005;63:184-6. 